\title{
Study on Bleaching Technology of Cotton Fabric with Sodium Percarbonate
}

\author{
Zhi $\mathrm{LI}^{1, \text { a }}$, Yanling $\mathrm{WANG}^{2, \mathrm{~b}}$ and Zhichao $\mathrm{WANG}^{3, \mathrm{c}}$ \\ ${ }^{1}$ Heilongjiang University Of Technology, Heilongjiang 158100, China \\ ${ }^{2}$ Heilongjiang University Of Technology, Heilongjiang 158100, China \\ ${ }^{3}$ Tianjin Polytechnic University, Tianjin 300160, China; \\ azhi021119@126.com, ${ }^{\mathrm{b}} 278358275 @ 163 . c o m,{ }^{\mathrm{c}} 403857886 @$ qq.com
}

\begin{abstract}
Bleach cotton fabric with sodium percarbonate solution. Analyse of the effect of the concentration of sodium percarbonate solution, bleaching time, bleaching temperature and the light radiation on the bleaching effect of fabric. The result shows that increasing concentrations of percarbonate,increasing the bleaching time, raising the bleaching temperature and the UV irradiation may whiten the cotton fabric.The most suitable conditions for the bleaching process is concentration of sodium percarbonate solution $6 \mathrm{~g} / \mathrm{L}$, bleaching temperature $80^{\circ} \mathrm{C}$ and bleaching time $60 \mathrm{~min}$.

Keywords: Cotton Fabric, Sodium Percarbonate, Bleaching
\end{abstract}

\section{Introduction}

Currently, the main method for bleaching cotton fabric is using sodium hypochlorite and hydrogen peroxide ${ }^{[1,2]}$. Sodium hypochlorite was gradually limited since it can produce more toxic chlorinated organic byproducts that can cause environmental pollution in process of bleaching. Althought the residue that bleached by hydrogen peroxide is non-toxic. But the strong alkaline of hydrogen will damage to cotton fabric and difficult to transport and storage due to peroxide will become liquid at room temperature.

In recent years, there has been many home and aboard reports about bleaching process of using sodium percarbonate to bleach cotton fabrics but not study on cotton knitted fabric and research with more details. Therefore, this article describes that using sodium percarbonate as decolorizer to bleach cotton knitted fabric, and focus on conditions of bleaching process such as sodium percarbonate concentration, bleaching time and influnce of bleaching effect of light radiation on the fabric. So it has important significance for developing new bleaching process and reducing environmental pollution. 


\section{Experimental Methods}

\subsection{Eagents and Materials}

Cotton fabric, single jersey (made in Tianjin China), Na2CO3, $\mathrm{H} 2 \mathrm{O} 2$ and $\mathrm{NaOH}(\mathrm{AR})$, efficient scouring agent(laboratory-made).

\subsection{Experimental Instrument}

Heated water bath(made in Medical equipment manufacturing plant Beijing), Datacolor3890 computer color measurement system( made in Datacolour USA), 125W UV radiation box(laboratory-made).

\subsection{Refining Process}

Formula of scouring the working fluid: $\mathrm{NaOH} 20 \%$,efficient scouring agent $2 \%$,bath ratio 1:30.

Accurately weigh a certain quality of cotton fabric and wet it. Put it into the working fluid according to the formula as described above and boil it for 2 hours under $100^{\circ} \mathrm{C}$ temperature, then take it out wash for 3 times with hot water which $90^{\circ} \mathrm{C}$ above. Finally, wash it again with cold water until free of alkali, and then dry it at room temperature to preparing for bleaching.

\subsection{Test Method of Bleaching Process and Whiteness}

Put the cotton fabric of accurate taken into sodium percarbonate working fluid which with a certain concentration. Keep the bath ratio as 1:30, and maintain the working fluid warm for a certain period at the specified temperature. Use color measurement system colour3890 to measure the cotton fabric CIE whiteness value.

\section{Results and Discussion}

\subsection{The Effect of the Concentration of Sodium Percarbonate Solution on the Whiteness CIE.}

Under the constant temperature of $80^{\circ} \mathrm{C}$, bleach the both fabric for two hours, and obverse the effect of sodium percarbonate on CIE whiteness, as shown in Fig.1. 


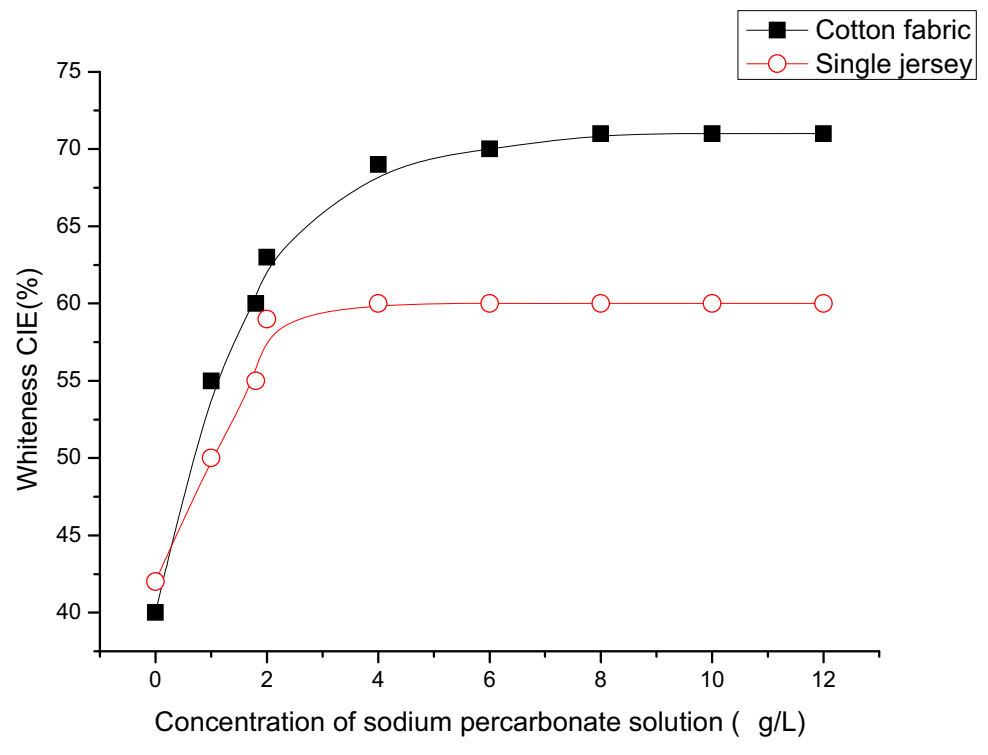

Fig.1 The effect of the concentration of sodium percarbonate solution on the whiteness CIE

Fig.1 indicated that CIE whiteness of the fabric rises faster, when the concentration of sodium percarbonate is between the $0-6 \mathrm{~g} / \mathrm{L}$. When it is more than $6 \mathrm{~g} / \mathrm{L}$, CIE whiteness of the fabric makes little change. Because the concentration of sodium percarbonate increases, the reactive oxygen species content in the solution increases. More pigment of fiber surface is oxidized and the CIE whiteness of the fabric becomes higher. Concentration of sodium percarbonate continue to increase would result in higher active oxygen in system, but did not enhance its capability of oxidation to pigment. In addition, In the same reaction conditions, Whiteness of cotton fabric is significantly higher than that of single jersey whiteness. This suggests that cotton fabric has higher adsorption capacity to active oxygen.

\subsection{The Effect of Temperature on the Whiteness CIE.}

Use the sodium percarbonate solution of $6 \mathrm{~g} / \mathrm{L}$ soaking cotton knit for two hours under the conditions of different temperatures. Tudy the effect of temperature on the fabric whiteness CIE. The result is shown in Figure 2. 


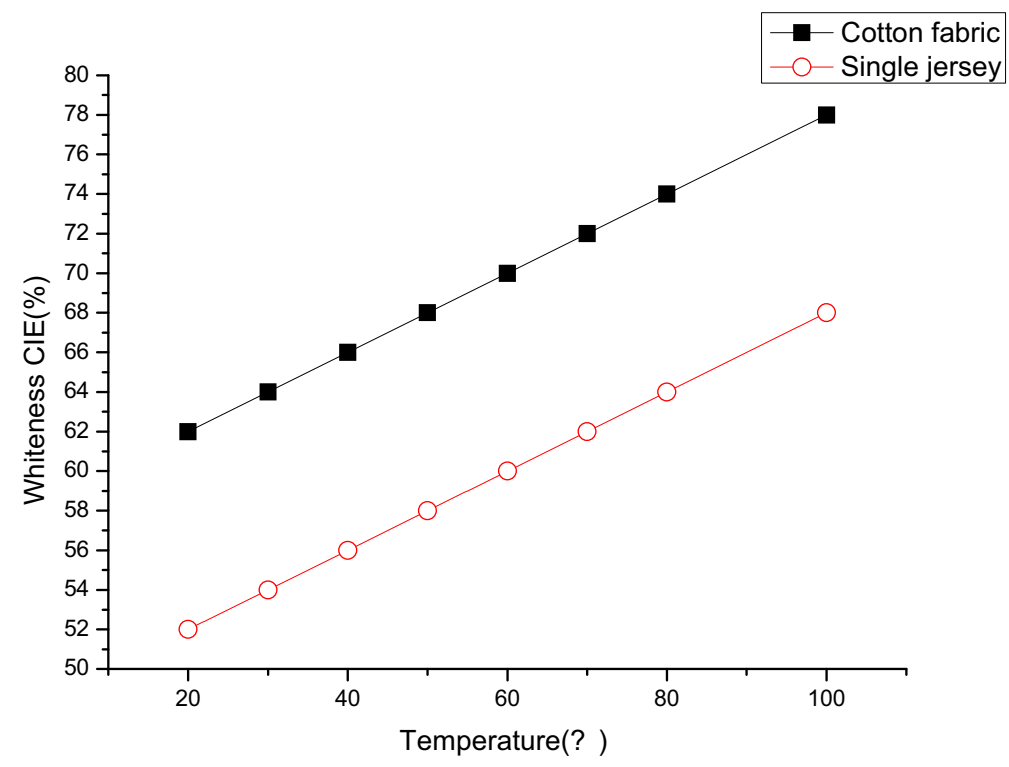

Figure 2 The effect of temperature on the whiteness CIE

Figure 2 shows that two kinds of fabric whiteness CIE have a linear relation with temperature. With the improvement of working liquid temperature, the fabric whiteness CIE scale up. Because the temperature increase leads to fast decompose of sodium percarbonate and redundantly produce reactive oxygen species.

\subsection{The Effect of Time on the Whiteness CIE.}

Use the sodium percarbonate solution of $6 \mathrm{~g} / \mathrm{L}$ soaking cotton knit for two hours under the condition of $80{ }^{\circ} \mathrm{C}$. Tudy the effect of time on the fabric whiteness CIE. The result is shown in figure 3 . 


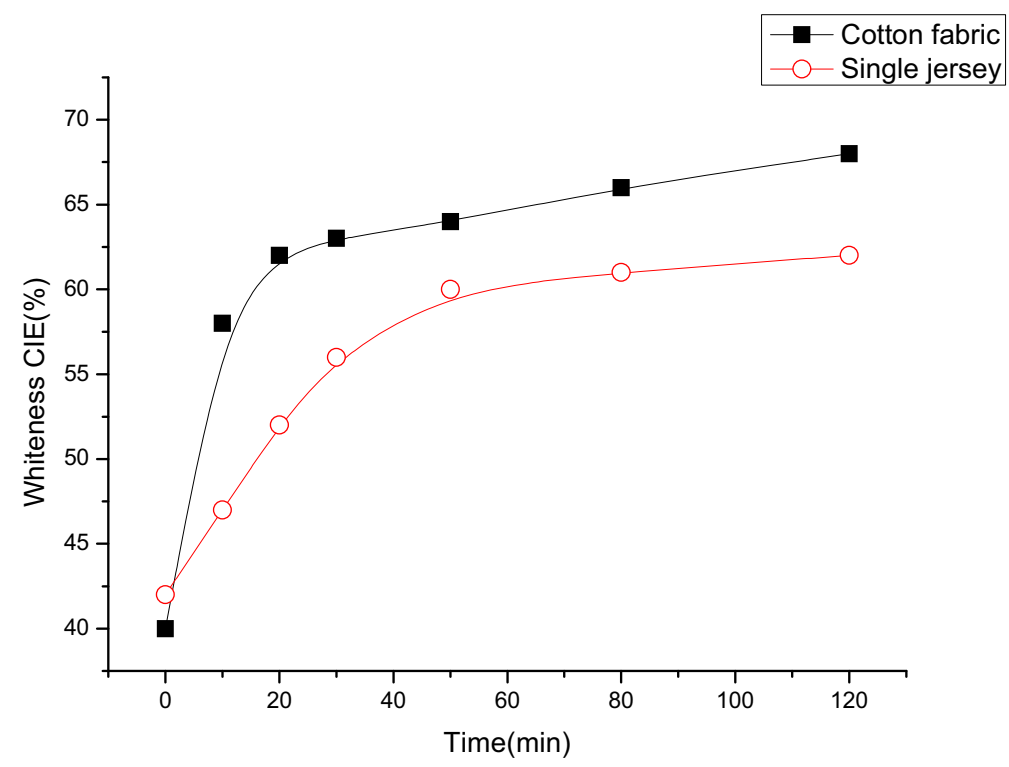

Figure 3 The effect of time on the whiteness CIE

Figure 3 shows that Fabric whiteness CIE increase significantly as bleaching time, at the beginning of the bleaching (in 60 minutes).But when time is more than 60 minutes, fabric whiteness CIE increase only slightly. It is because that the bleaching time makes the system of active oxygen concentration increased and it is good for fabric bleaching. From the point of view of saving energy, suitable bleaching time should be set to $60 \mathrm{~min}$,in the use of sodium percarbonate bleaching cotton knitted fabric.

\subsection{The Effect of Light Radiation on the Whiteness CIE.}

In order to study the effect of light radiation, the fabric was immersed in sodium percarbonate solution with $5 \mathrm{~min}$. And then divide it into two parts. In the light reactor, the first group was treated with a 60 min under normal temperature(AT-E Process). The second group was treated with a $60 \mathrm{~min}$ under $80{ }^{\circ} \mathrm{C}$ (HT-E Process). Finally, determine the whiteness of two kinds of fabrics as shown in figure 4. 


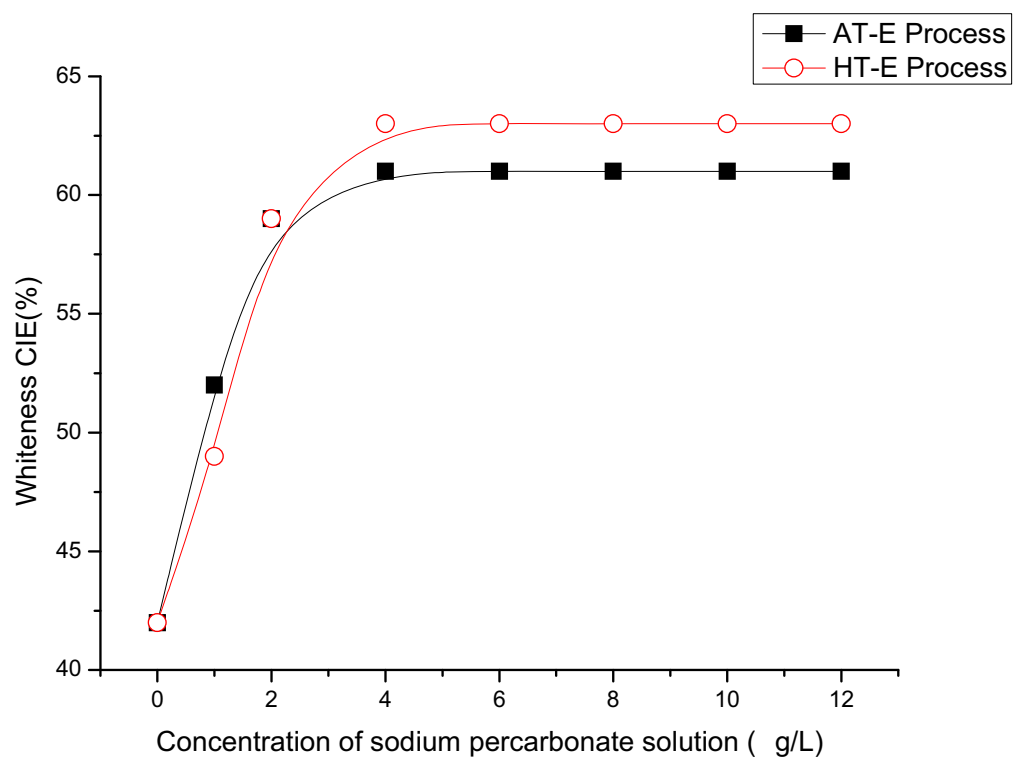

Figure 4 The effect of light radiation on the whiteness CIE

Figure 4 shows that with the increase of solution concentration of sodium percarbonate, the whiteness of the fabric is gradually increasing. When the concentration of sodium percarbonate was less than $2 \mathrm{~g} / \mathrm{L}$, the whiteness of two kinds of bleached fabrics is almost the same. When the sodium percarbonate concentration continues to increase, the whiteness of bleached fabrics using high temperature impregnation process (HT-E) is better then the whiteness of bleached fabrics using ambient temperature impregnation process (AT-E). But there is little difference between the two. It is proved that under normal temperature, UV has a significant effect on the release of reactive oxygen species. The reason may be that the sodium percarbonate in the solution absorbs ultraviolet light.It reduces the activation energy of decomposition reaction and increases the release of reactive oxygen species. And it can improve the whiteness of bleached fabrics. This further shows that sodium percarbonate can be used to bleach cotton fabrics under the light irradiation conditions. This process is very important for energy saving.

\section{Conclusion}

With the increase of the concentration of sodium percarbonate, the whiteness of bleached cotton fabric is significantly improved. When the concentration of sodium percarbonate was over $6 \mathrm{~g} / \mathrm{L}$, there was no obvious change in whiteness. In the same conditions, the degree of whiteness of the white cotton fabric was significantly higher than that of single jersey.

The whiteness of cotton fabric increased linearly with the increase of bleaching temperature. However, under the condition of sodium percarbonate concentration of $6 \mathrm{~g} / \mathrm{L}$, the bleaching time is more suitable for $60 \mathrm{~min}$ when the solution temperature is 80 .

Ultraviolet ray irradiation can significantly improve the whiteness of bleached cotton fabrics. And it makes cotton fabric bleached at room temperature. 


\section{References}

1. Hui Li,Zuquan Jin. A study on the application of sodium percarbonate in the scouring and bleaching of cotton fabrics. J .Textile auxiliaries. 1996, 13 (6) : 15, 19-20.

2. OUCHI A. Chlorine free bleaching of fabrics. J .International Textile Leader. 2007 (4) : 64-68. 